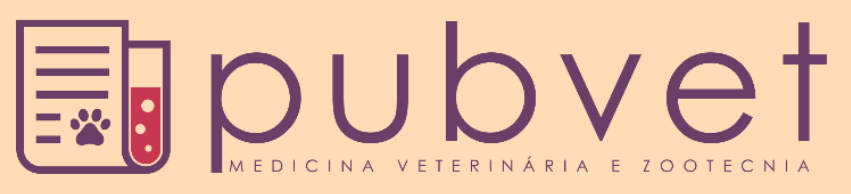

https://doi.org/10.31533/pubvet.v14n5a562.1-7

\title{
Erliquiose monocítica canina recidivante: relato de caso
}

\author{
Vinícius Macedo Padilha ${ }^{1}$, Martha Bravo Cruz Piñeiro ${ }^{2}{ }^{\ominus}$, Bruno Cabral Chagas $^{3}{ }^{\bullet}$, Julia \\ Somavilla Lignon ${ }^{4 *}$, Márcia de Oliveira Nobre ${ }^{5} \bullet$ \\ ${ }^{I}$ Médico Veterinário pela Universidade Federal de Pelotas, Pelotas, Rio Grande do Sul, Brasil. \\ ${ }^{2}$ Mestranda do Programa de Pós-graduação em Veterinária da Universidade Federal de Pelotas, Pelotas, Rio Grande do Sul, Brasil. \\ ${ }^{3}$ Doutorando do Programa de Pós-graduação em Veterinária da Universidade Federal de Pelotas, Pelotas, Rio Grande do Sul, Brasil. \\ ${ }^{4}$ Médica Veterinária pela Universidade Federal de Pelotas, Pelotas, Rio Grande do Sul, Brasil. \\ ${ }^{5}$ Professora da Faculdade de Veterinária, Universidade Federal de Pelotas, Pelotas, Rio Grande do Sul, Brasil. \\ *Autor para correspondência, E-mail: julialignon@gmail.com
}

Resumo. A Erliquiose Monocítica Canina é uma doença causada pela bactéria intracitoplasmática obrigatória Erlichia canis, tendo como vetor o carrapato dos cães Rhipicephalus sanguineus. Possui curso clínico baseado em apresentações aguda, subclínica e crônica, variando a intensidade dos sinais, podendo cursar com anemia, depressão e em casos crônicos depleção. Apresenta distribuição mundial, porém mesmo com o vetor intensamente presente no Rio Grande do Sul, o número de relatos clínicos científicos é muito baixo. O objetivo deste trabalho foi evidenciar um relato de caso de Erliquiose Monocítica Canina em um cão no estado do Rio Grande do Sul, considerado não endêmico para a enfermidade. Um cão, Shih Tzu, fêmea, atópico, cinco anos de idade foi atendida com queixa de falta de apetite e apatia. No hemograma apresentou intensa anemia hipocrômica macrocítica. O esfregaço foi positivo para Erlichia sp. Foi instituído um tratamento com doxiciclina com posterior estabilização do quadro. Três meses após, o animal voltou a apresentar a mesma sintomatologia de apatia e falta de apetite, sendo diagnosticada com anemia normocítica hipercrômica e hematócrito de 5\%. Foi instituída imediata transfusão sanguínea e novo tratamento com doxiciclina por 15 dias, sendo constatada melhora do quadro após este período. Este trabalho ressaltou a importância do correto tratamento e diagnóstico dessa enfermidade, realizado através dos sinais clínicos, identificação dos vetores da doença, os métodos laboratoriais disponíveis bem como este relato incorpora-se a bibliografia científica sobre a Erliquiose Monocítica Canina, auxiliando veterinários clínicos devido ao baixo acervo sobre a doença na região Sul.

Palavras chave: anemia, cães, doxiciclina, Erlichia sp

\section{Recurrent canine monocytic ehrlichiosis: case report}

bstract. Canine Monocytic Ehrlichiosis is a disease caused by the obligate intracytoplasmic bacterium Erlichia canis, having as vector the tick of the dogs Rhipicephalus sanguineus. It has a clinical course based on acute, subclinical and chronic presentations, varying the intensity of the signs, being able to attend with anemia, depression and in chronic cases depletion. It has a worldwide distribution, but even with the vector intensely present in Rio Grande do Sul, the number of scientific clinical reports are very low. The objective of this work was to report a case of Canine Monocytic Ehrlichiosis in a dog in the state of Rio Grande do Sul. A female, 5-year-old, atopic, Shih Tzu was treated with complaint of lack of appetite and apathy. The hemogram showed intense macrocytic hypochromic anemia. The smear was positive for Erlichia sp. A treatment with doxycycline was instituted with subsequent stabilization of the condition. Three months later, the animal returned to the same symptomatology of apathy and lack of appetite, being diagnosed with hyperchromic normocytic anemia and hematocrit of $5 \%$. 
Immediate blood transfusion and a new treatment with doxycycline of 15 days were instituted, being an improvement of the picture after this period. This work underscored the importance of correct diagnosis and treatment as well as incorporating the scientific literature on Canine Monocytic Ehrlichiosis in Rio Grande do Sul.

Keywords: anemia, dogs, doxycycline, Erlichia sp

\section{Erliquiosis monocítica canina recidivante: relato de caso}

Resumen. La Erliquiosis Monocítica Canina es una enfermedad causada por la bacteria intracitoplasmática obligatoria Erlichia canis, teniendo como vector la garrapata de los perros Rhipicephalus sanguineus. Tienen un curso clínico basado en presentaciones agudas, subclínicas y crónicas, variando la intensidad de las señales, pudiendo cursar con anemia, depresión y en casos crónicos depleción. Presenta distribución mundial, pero incluso con el vector intensamente presente en Rio Grande do Sul, el número de relatos clínicos es muy bajos. El objetivo de este trabajo fue evidenciar un relato de caso de Erliquiosis Monocítica Canina en un perro en el estado de Rio Grande do Sul, considerado no endémico para la enfermedad. Un perro, Shih Tzu, hembra, atópico, cinco años de edad fue atendida con queja de falta de apetito y apatía. En el hemograma presentó una intensa anemia hipocrómica macrocítica. El frotis fue positivo para Erlichia sp. Se estableció un tratamiento con doxiciclina con posterior estabilización del cuadro. Tres meses después, el animal volvió a presentar la misma sintomatología de apatía y falta de apetito, siendo diagnosticada con anemia normocítica hipercrómica y hematócrita del 5\%. Se instituyó inmediata transfusión sanguínea y nuevo tratamiento con doxiciclina por 15 días, siendo constatada mejora del cuadro después de este período. Este trabajo resaltó la importancia del correcto tratamiento y diagnóstico de esta enfermedad, realizado través de los signos clínicos, identificación de los vectores de la enfermedad, los métodos de laboratorio disponibles bien como este relato se incorpora a la bibliografía científica sobre la Erliquiosis Monocítica Canina, auxiliando veterinarios clínicos debido al bajo acervo sobre la enfermedad en la región Sur.

Palabras clave: anemia, perros, doxiciclina, Erlichia sp

\section{Introdução}

Nos últimos anos cada vez são mais frequentes os relatos de infestações de cães por carrapatos, que podem transmitir uma variedade de agentes patogênicos, tanto para os animais como para o homem. Um dos principais agentes é a Ehrlichia sp., hemoparasito intracelular obrigatório de células mononucleares e nos cães ocasionam a doença Erliquiose Monocítica Canina (EMC) (Isola et al., 2012; Mendonça et al., 2005; Silva et al., 2013).

A doença tem distribuição mundial, com alta prevalência em países tropicas e subtropicais; porém, com ocorrência de casos em países de clima temperado em detrimento a enorme adaptabilidade do principal vetor, Rhipicephalus sanguineus, a diferentes regiões e climas (Dantas-Torres, 2010). A espécie do agente encontrado no país é a Erlichia canis com endemicidade em aglomerados populacionais urbanos devido ao acentuado aparecimento do carrapato vetor, que possui hábito de alojar-se em locais que ofereçam de um micro clima com alta umidade e temperatura média, como pátios com gramados, entulhos e paredes de cimento, sendo que a presença deste na pele do animal, dependendo do seu estágio de evolução, por vezes não é notada e pode figurar como o diferencial da avaliação na anamnese pelo médico veterinário. A transmissão de Erlichia sp. pode ocorrer também por transfusões sanguíneas de um animal positivo para um suscetível (García et al., 2007; Silva et al., 2013).

Os sinais clínicos da EMC podem apresentar-se em fases aguda, subclínica ou crônica, sendo a trombocitopenia bem como sinais envolvendo a depleção sanguínea em geral, comum a todas as fases (McClure et al., 2010). Considerando a diversa apresentação clínica da EMC, o fato de seu diagnóstico ser de triagem em atendimentos, pela doença ser endêmica em regiões urbanas e mesmo assim existir baixo acervo bibliográfico científico sobre a doença na região sul do Brasil, objetivamos evidenciar um relato de 
caso clínico de EMC em um cão no Sul do estado do Rio Grande do Sul, considerado não endêmico para a enfermidade.

\section{Material e métodos}

Foi atendido um canino, fêmea, da raça Shih Tzu, com cinco anos de idade, $5 \mathrm{~kg}$, com a queixa de apatia intensa e falta de apetite na cidade de Caxias do Sul. A paciente era castrada, com vacinação e vermifugação em dia, sem acesso livre a rua e sem contato com outros animais, alimentação comercial adequada à idade e a raça e acesso livre a água. Com histórico de dermatite, a fêmea era tratada periodicamente com o imunossupressor prednisolona. Através do relato do tutor, evidenciou-se que em uma viagem realizada ao litoral, foi notada a presença de um carrapato e o mesmo retirado manualmente da pele do canino. Ao exame clínico a mesma apresentava sinais de apatia, mucosas ictéricas, taquipnéia, taquicardia e pirexia. Foram solicitados exames complementares e hemograma completo.

\section{Resultados}

No exame ultrassonográfico foi evidenciada hepatomegalia discreta e renomegalia. O hemograma completo apresentou leucocitose por neutrofilia com desvio a esquerda (Tabela 1), contagem de reticulócitos que evidenciou anemia macrocítica hipocrômica, policromasia intensa, corpúsculos de Howell-Jolly e contagem absoluta de reticulócitos de 340.750/ $\mu 1$ com regeneração máxima (Figuras 1 e 2). O esfregaço sanguíneo realizado evidenciou a presença de mórulas de Erlichia sp. em inclusões citoplasmáticas (Figura 3).

Tabela 1. Resultados dos hemogramas (série branca), realizados durante a apresentação clínica e recidiva de Erliquiose Monocítica Canina, ambos com avaliação pré e pós-tratamento

\begin{tabular}{lccccc}
\hline & \multicolumn{2}{c}{ Caso clínico } & \multicolumn{2}{c}{ Recidiva } \\
\hline Hemograma & Valores de referência & Exame & Revisão & Exame & Revisão \\
\hline Leucócitos & $8-16$ milhões/mm3 & $32.800^{*}$ & $24.100^{*}$ & $34.700^{*}$ & $27.200^{*}$ \\
Linfócitos & $10-26 \%$ & $7^{*}$ & $6^{*}$ & $5^{*}$ & $7^{*}$ \\
Bastonetes & $0-1 \%$ & $19 *$ & - & $17^{*}$ & $2^{*}$ \\
Segmentados & $55-80 \%$ & 69 & - & 68 & 89 \\
Eosinófilos & $1-9 \%$ & 1 & $9,2^{*}$ & $00^{*}$ & $0^{*}$ \\
Monócitos & $1-6 \%$ & 3 & - & $8^{*}$ & 2 \\
Proteínas Totais & $6-8 \mathrm{~g} / \mathrm{dL}$ & - & - & 7,2 & $8,1^{*}$ \\
Plaquetas & $200-500 / \mathrm{mm}^{3}$ & 237 & 318 & $168^{*}$ & $706^{*}$ \\
\hline
\end{tabular}

*Parâmetros alterados em relação aos valores de referência.

Sob a confirmação clínica de hemoparasitose por Erlichia sp. através do esfregaço sanguíneo, foi recomendada administração de doxiciclina, $1 / 4$ comprimido, a cada 12 horas, via oral durante 24 dias seguidos. Após 24 dias de tratamento foi realizada uma segunda avaliação hematológica do animal, demonstrando estabilização do quadro clínico e normalização dos resultados hematológicos (Figuras 1 e 2).

Três meses após o primeiro atendimento o paciente retornou ao veterinário com os mesmos sintomas e sinais clínicos ao exame físico. Considerando uma possível recidiva foi efetuado novamente o hemograma e esfregaço sanguíneo corado proveniente de sangue periférico. Os exames demonstraram anemia normocítica normocrômica severa, eritrócitos fantasmas, anisocitose, policromasia, esferócitos (Figura 1), leucocitose por neutrofilia com desvio a esquerda, neutrófilos tóxicos e trombocitopenia (Tabela 1). O diagnóstico de recidiva de Erliquiose foi confirmado pela presença de corpúsculos intracitoplasmáticos (mórulas) de Ehrlichia sp. em neutrófilos.

Diante do diagnóstico de Erliquiose Monocítica Canina em apresentação crônica e a anemia severa, realizou-se quatro transfusões sanguíneas, intercaladas, juntamente com a administração de doxiciclina2 $5 \mathrm{mg} / \mathrm{Kg}$, a cada 12 horas, via oral, por 15 dias consecutivos bem como tratamento de suporte. Após o tratamento foi realizado novo hemograma completo (Tabela 1, Figura 1 e 2), onde se observou a recuperação da anemia, com resquícios de sinais de macrocitose, hipocromia, leucocitose por neutrofilia 
com desvio a esquerda, trombocitose e contagem absoluta de reticulócitos de 183.625/ $\mu$, indicando anemia regenerativa com liberação discreta a moderada.

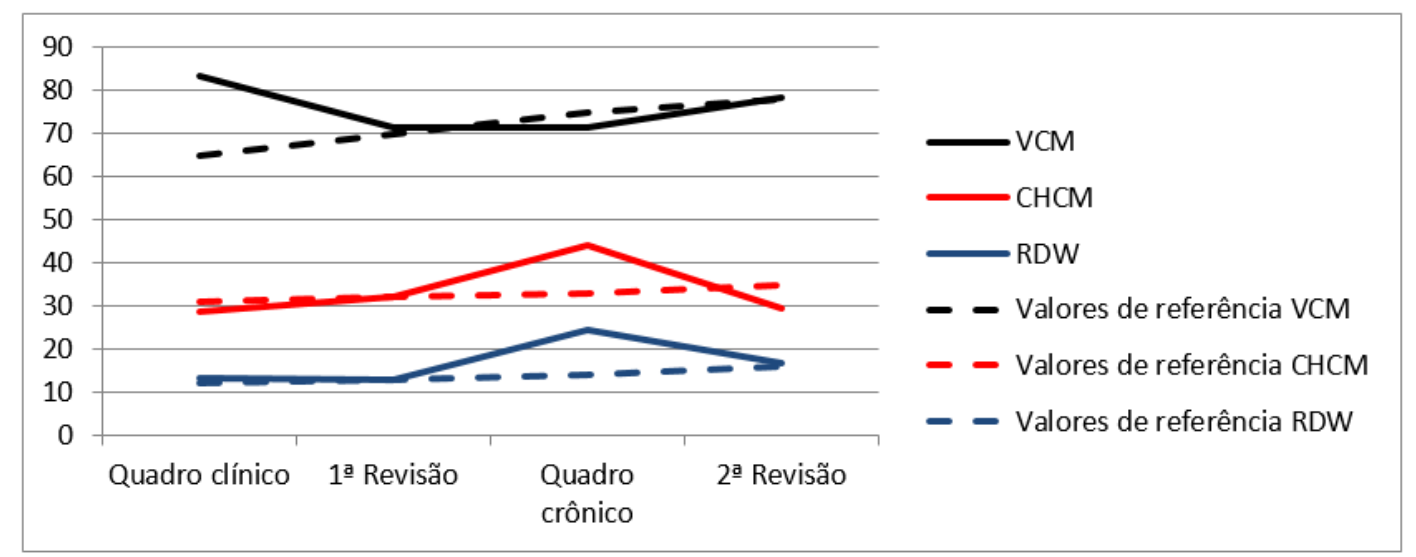

Figura 1. Dados de Volume Corpuscular Médio (VCM), concentração de hemoglobina corpuscular média $(\mathrm{CHCM})$, red cell distribution width (RDW) e os valores de referência de cada parâmetro.

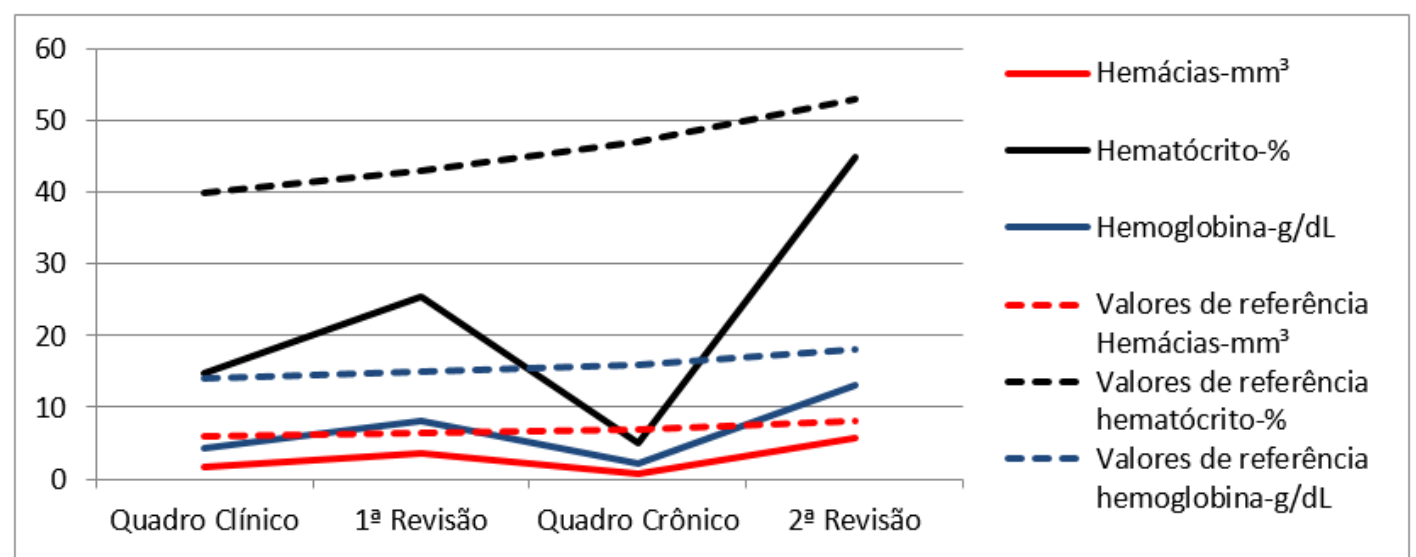

Figura 2. Valores de Hemácias $\left(\mathrm{mm}^{3}\right)$, hematócrito (\%), hemoglobina (g/dL) e os padrões de referência de cada parâmetro.

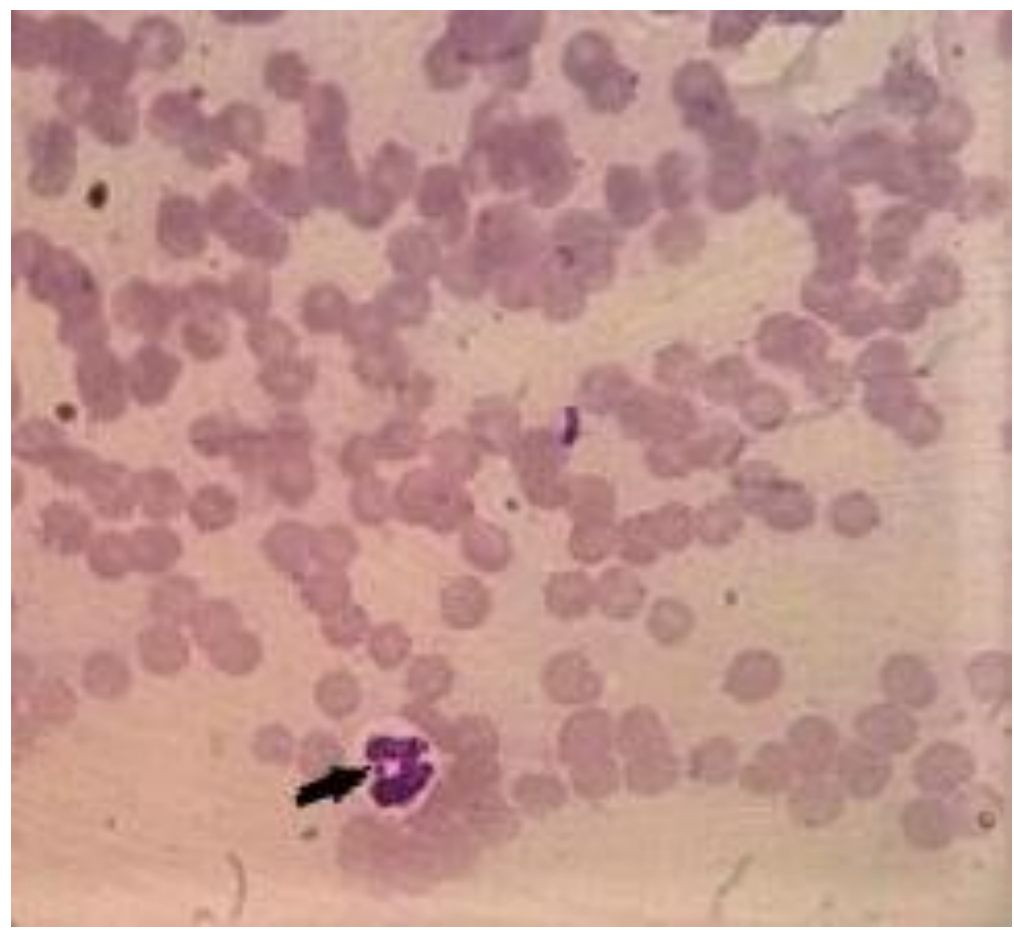

Figura 3. Mórula de Erlichia sp. (identificada com seta) em inclusão citoplasmática de leucócito em esfregaço sanguíneo corado. 


\section{Discussão}

A epidemiologia sobre a infecção por E. canis no Brasil, assim como no Rio Grande do Sul, vem sendo estudada, envolvendo a clínica da doença, seja avaliando a idade, sexo e raça dos cães e até mesmo a presença de carrapatos, na tentativa de se estabelecer fatores de risco para a doença (Dagnone et al., 2002). Países vizinhos como o Uruguai e o Paraguai assemelham-se aos estados do sul por relatarem baixas incidências da doença (Day, 2011). O número de casos positivos de erliquiose está diretamente associado à distribuição do vetor encontrado principalmente em regiões de clima tropical, sendo que essa diferença é acentuada pela maior ou menor quantidade de carrapatos, a origem dos animais ou a sensibilidade dos diferentes testes de diagnósticos (Carlos et al., 2007), sendo de extrema importância a avaliação das características da doença bem como de sua epidemiologia como análise de triagem clínica.

Os sinais clínicos apresentados, como apatia, anemia e enfraquecimento relacionam-se tanto na fase aguda quanto na reagudização em fase crônica com a capacidade de reprodução exponencial do parasito em células sanguíneas e ainda a sua capacidade em passar despercebido pela vigília do sistema imune com estratégias de sobrevivência no hospedeiro, como o impedimento da fusão de lisossomos, as inclusões citoplasmáticas (mórulas) o que favorece a sua mantença reprodução (Alves et al., 2013). A condição constante de leucocitose mesmo após os dois quadros de melhora das condições gerais hematológicas expressas nos hemogramas realizados nos atendimentos, deriva da condição da resposta imunológica do organismo do paciente frente à infecção por Erlichia sp. mesmo o animal possuindo uma condição de atopia com administração frequente de prednisolona, a qual leva a imunossupressão (Olivry et al., 2002).

O diagnóstico realizado via esfregaço sanguíneo é a prática de rotina laboratorial mais recorrente e utilizada, com visualização das mórulas no citoplasma de neutrófilos e monócitos. Mesmo bem executada, esta técnica possui uma taxa de observação do parasito entre 4 a $6 \%$, sendo necessário e quando possível teste de confirmação através de outras técnicas (Sainz et al., 2015), o que não foi realizado neste caso devido o achado das mórulas no esfregaço e eficácia do tratamento frente aos sinais clínicos.

Carlos et al. (2007) relatam que o microrganismo se multiplica dentro das células mononucleares circulantes e dos tecidos fagocitários mononucleares do fígado, baço e linfonodos, levando à linfadenomegalia e à hiperplasia do fígado e/ou do baço, demonstrados discretamente no atendimento da fase aguda da doença (Machado, 2004). O tratamento recomendado nesse caso clínico, tanto na manifestação aguda da doença quanto na apresentação crônica foi a doxiciclina, sendo esta a droga de eleição para o tratamento da erliquiose por possuir uma rápida absorção quando administrada por via oral, com distribuição ampla pelos tecidos e efeito superior ao cloridrato de tetraciclina e a oxitetraciclina (Davoust, 1993; Little, 2010) e mesmo com descrição de uso (Sainz et al., 2000), não é recomendado a utilização de dipropionato de imidocarb pela ineficiente ação frente à Erlichia sp. (Eddlestone et al., 2006; Matthewman et al., 1998), somente em casos de co-infecção por Babesia canis. O tratamento pode durar de 3 a 4 semanas nos casos agudos e até oito semanas nos casos crônicos (Woody \& Hoskins, 1991).

Nas duas manifestações da doença, aguda e crônica, o tratamento foi efetivo em virtude da estabilização dos parâmetros sanguíneos, porém é notável o papel do carrapato como vetor nesse caso, visto que apenas um exemplar encontrado parasitando o paciente possivelmente tenha levado ao quadro de erliquiose. Uma avaliação quanto à presença de carrapatos jovens e adultos nos animais mostra-se de suma importância, pois em qualquer estágio o mesmo pode transmitir a Erlichia sp. (Almeida et al., 2012).

\section{Conclusões}

Este trabalho relatou um caso de Erliquiose Monocítica Canina em um cão no estado do Rio Grande do Sul, afirmando a importância do diagnóstico da doença desde a identificação dos vetores da doença bem como das técnicas laboratoriais disponíveis, apresentação e desenvolvimento a partir de sintomatologia nos cães, sendo de suma importância o relato destes casos para os clínicos veterinários da região sul pelo ínfimo acervo técnico dos mesmos.

\section{Referências bibliográficas}

Almeida, A. B. P. F., Paula, D. A. J., Dahroug, M. A. A., Freitas, A. G., Silva, J. N., Dutra, V., Nkazato, 
L., \& Sousa, V. R. F. (2012). Ehrlichia canis e Anaplasma platys em carrapatos de cães de Cuiabá, Mato Grosso. Semina: Ciências Agrárias, 33(3), 1123-1126.

Alves, R. N., Levenhagen, M. A., Levenhagen, M., Rieck, S. E., Labruna, M. B., \& Beletti, M. E. (2013). A manutenção de Ehrlichia canis em macrófagos é dependente da evasão lisossomal. Ars Veterinaria, 29(4), 5. https://doi.org/http://dx.doi.org/10.15361/2175-0106.2013v29n4p5.

Carlos, R. S. A., Neta, M., Elza, S., Spagnol, F. H., Oliveira, L. L. S., Brito, R. L. L., Albuquerque, G. R., \& Almosny, N. R. P. (2007). Freqüência de anticorpos anti-Erhlichia canis, Borrelia burgdorferi e antígenos de Dirofilaria immitis em cães na microrregião Ilhéus-Itabuna, Bahia, Brasil. Revista Brasileira de Parasitologia Veterinária, 16(3), 117-120. https://doi.org/https://doi.org/10.1590/S1984-29612007000300001.

Dagnone, A. S., Trapp, S. M., Jojima, F. S., Amude, A. M., Morais, H. A. S., Freire, R. L., \& Vidotto, O. (2002). Avaliação Soroepidemiológica da infecção por Ehrlichia canis, Dirofilaria immitis e Borrelia burgdorferi em cães de uma população hospitalar. XII Congresso Brasileiro de Parasitologia Veterinária, Rio de Janeiro.

Dantas-Torres, F. (2010). Biology and ecology of the brown dog tick, Rhipicephalus sanguineus. Parasites \& Vectors, 3(1), 26. https://doi.org/https://doi.org/10.1186/1756-3305-3-26.

Davoust, B. (1993). Canine ehrlichiosis. Point Vétérinaire, 25(151), 43-51.

Day, M. J. (2011). One health: the importance of companion animal vector-borne diseases. Parasites \& Vectors, 4(49), 1-6.

Eddlestone, S. M., Neer, T. M., Gaunt, S. D., Corstvet, R., Gill, A., Hosgood, G., Hegarty, B., \& Breitschwerdt, E. B. (2006). Failure of imidocarb dipropionate to clear experimentally induced Ehrlichia canis infection in dogs. Journal of Veterinary Internal Medicine, 20(4), 840-844. https://doi.org/https://doi.org/10.1111/j.1939-1676.2006.tb01795.x.

García, M. E., Moissant, E., Pérez, A., Quijada, J., Simoes, D., \& García, H. (2007). Comportamiento natural de las fases no parasíticas de Rhipicephalus sanguineus (Latreille, 1806)(Acari: Ixodidae) en un bioterio canino de Venezuela. Revista Científica, 17(6), 566-571.

Isola, J. G. M. P., Cadioli, F. A., \& Nakage, A. P. (2012). Erliquiose canina-revisão de literatura. Revista Científica Eletrônica de Medicina Veterinária, 18, 1-11.

Little, S. E. (2010). Ehrlichiosis and anaplasmosis in dogs and cats. Veterinary Clinics: Small Animal Practice, 40(6), 1121-1140.

Machado, R. Z. (2004). Erliquiose canina. Revista Brasileira de Parasitologia Veterinária, 13, 53-57.

Matthewman, L. A., Raoult, D., Brouqui, P., \& Kelly, P. J. (1998). Lack of susceptibility of Ehrlichia canis to imidocarb dipropionate in vitro. Journal of the South African Veterinary Association, 69(2), $55-56$.

McClure, J. C., Crothers, M. L., Schaefer, J. J., Stanley, P. D., Needham, G. R., Ewing, S. A., \& Stich, R. W. (2010). Efficacy of a doxycycline treatment regimen initiated during three different phases of experimental ehrlichiosis. Antimicrobial Agents and Chemotherapy, 54(12), 5012-5020. https://doi.org/http//:doi.org/10.1128/AAC.01622-09.

Mendonça, C. S., Mundim, A. V., Costa, A. S., \& Moro, T. V. (2005). Erliquiose canina: Alterações hematológicas em cães domésticos naturalmente infectados. Bioscience Journal, 21(1), 167-174.

Olivry, T., Rivierre, C., Jackson, H. A., Murphy, K. M., Davidson, G., \& Sousa, C. A. (2002). Cyclosporine decreases skin lesions and pruritus in dogs with atopic dermatitis: a blinded randomized prednisolone-controlled trial. Veterinary Dermatology, 13(2), 77-87. https://doi.org/https://doi.org/10.1046/j.1365-3164.2002.00283.x.

Sainz, Á., Roura, X., Miró, G., Estrada-Peña, A., Kohn, B., Harrus, S., \& Solano-Gallego, L. (2015). Guideline for veterinary practitioners on canine ehrlichiosis and anaplasmosis in Europe. Parasites \& Vectors, 8(1), 75. https://doi.org/https://doi.org/10.1186/s13071-015-0649-0. 
Sainz, A., Tesouro, M. A., Amusategui, I., Rodriguez, F., Mazzucchelli, F., \& Rodriguez, M. (2000). Prospective comparative study of 3 treatment protocols using doxycycline or imidocarb dipropionate in dogs with naturally occurring ehrlichiosis. Journal of Veterinary Internal Medicine, 14(2), 134139. https://doi.org/https://doi.org/10.1111/j.1939-1676.2000.tb02226.x.

Silva, M. V. M., Fernandes, R. A., Nogueira, J. L., \& Ambrósio, C. E. (2013). Erliquiose canina: revisão de literatura. Arquivos de Ciências Veterinárias e Zoologia Da UNIPAR, 14(2), 139-143.

Woody, B. J., \& Hoskins, J. D. (1991). Ehrlichial diseases of dogs. Veterinary Clinics of North America: Small Animal Practice, 21(1), 75-98.

Recebido: 19 de dezembro, 2019.

Aprovado: 7 de fevereiro, 2020.

Disponível online: 1 maio, 2020.

Licenciamento: Este artigo é publicado na modalidade Acesso Aberto sob a licença Creative Commons Atribuição 4.0 (CC-BY 4.0), a qual permite uso irrestrito, distribuição, reprodução em qualquer meio, desde que o autor e a fonte sejam devidamente creditados. 